Research Paper

\title{
Different Susceptibilities of Human Melanoma Cell Lines to G2/M Blockage and Cell Death Activation in Response to the Estrogen Receptor $\beta$ agonist LY500307
}

\author{
Giada Pontecorvi ${ }^{1}$, Maria Bellenghi ${ }^{1}$, Sabrina Tait ${ }^{1}$, Valentina Tirelli ${ }^{2}$, Paola Matarrese ${ }^{1}$, Gianfranco \\ Mattia $^{1}{ }^{\bowtie}$, Alessandra Carè ${ }^{{ }^{\bowtie \#}}$ and Rossella Puglisi1 ${ }^{\#}$ \\ 1. Center for Gender-Specific Medicine, Istituto Superiore di Sanità, Rome, Italy \\ 2. Core facilities, Istituto Superiore di Sanità, Rome, Italy \\ \# Equal senior authorship \\ $\triangle$ Corresponding authors: A. Carè and G. Mattia, Istituto Superiore di Sanità, Viale Regina Elena 299, 00161 Rome, Italy. E-mail: alessandra.care@iss.it and \\ gianfranco.mattia@iss.it \\ (C) The author(s). This is an open access article distributed under the terms of the Creative Commons Attribution License (https://creativecommons.org/licenses/by/4.0/). \\ See http:/ /ivyspring.com/terms for full terms and conditions.
}

Received: 2021.07.27; Accepted: 2021.11.04; Published: 2022.03.06

\begin{abstract}
Background: Gender differences in melanoma incidence, metastasis formation and disease progression are increasingly evident in epidemiological studies, with women showing significantly better survival than men. Among factors possibly underlying the disparities, sex hormones seem to play a key role. Nonetheless, functional mechanisms are still unclear, except for the antitumor ability of Estrogen Receptor (ER) $\beta$, whose expression determination has often been suggested for melanoma prognosis. In this study, we aimed at evaluating the molecular mechanisms and functional effects associated with ER $\beta$ signaling by using its agonist LY500307.

Methods: We evaluated the antitumor effect of the specific synthetic ER $\beta$ agonist LY500307 on some human melanoma cell lines, selected for different genetic background, expression levels of ERs and tumor progression. The expression of $\alpha$ and $\beta$ estrogen receptors was investigated taking advantage of The Cancer Genome Atlas database and confirmed on some selected melanoma cell lines. The biological effects of LY500307 were determined in vitro looking at melanoma cell proliferation, cell cycle profiles and migration demonstrating by western blot the involvement of some pathway specific markers. The LY500307-dependent induction of cell death was also analyzed by flow cytometry and western blot analysis of caspase 3 and poly adenosine diphosphate-ribose polymerase (PARP).

Results: A significant decrease in the expression of both ERs, even more pronounced for ERa, has been found in patients with metastatic NRAS mutation. Treatment with LY500307 significantly reduced the proliferation of melanoma cells showing a cell cycle arrest at the G2/M boundary phase and promoting apoptosis with different sensitivities associated with disease stage and mutation. Indeed, the ER $\beta$ agonist affects melanoma migration, inducing a reversion of the epithelial-mesenchymal transition, more evident in a low aggressive primary melanoma cell line.

Conclusion: These results demonstrate the capability of LY500307 to reduce melanoma malignancy, counteracting cell viability and dissemination, overall suggesting a possible future use of LY500307 in personalized combined therapy.
\end{abstract}

Key words: melanoma, LY500307, Estrogen Receptors, cell cycle, apoptosis.

\section{Introduction}

Cutaneous melanoma is the most aggressive and deadly type of skin cancer, mainly due to its high heterogeneity and remarkable propensity for metastatic spreading. Epidemiological data show sex divergences in both melanoma incidence and mortality rate, being men the most affected [1]. 
Searching for factors responsible for gender differences, a pivotal role of sex hormones has been suggested, indicating melanoma as a hormone-related cancer. Obviously, the involvement of other mechanisms cannot be excluded, leaving still unresolved the actual relevance of the hormonal influence on melanoma onset and progression. Sex influence on melanoma incidence and progression depends on both the hormonal concentration in the bloodstream and the expression of its receptors. Indeed, 17 $\beta$-estradiol, the main active circulating form of estrogen, plays an important protective role by strengthening the immune system [2]. Unfortunately, its therapeutic application is severely limited by enormous risks of developing other types of cancer, especially in women. Furthermore, as estrogen ability to bind with a similar affinity both estrogenic receptor $\alpha$ (ERa) and $\beta$ (ER $\beta$ ), pro- and antiproliferative respectively, it can evoke opposite effects in melanoma [3]. Therefore, determining the relative expression of each ER, more than their absolute amounts, could better predict estrogenic stimulus outcomes in a given tissue.

Looking at ERa, its presence or absence in both primary and metastatic melanoma specimens remains an unresolved issue [4]. However, the presence of ERa mRNA and the increase of epigenetic control of its promoter with disease progression have been demonstrated $[5,6]$. Conversely, several studies conducted on human melanoma tissues agree on ER $\beta$ decreased expression compared to healthy perilesional skin and, more generally, on the presence of an inverse correlation between ER $\beta$ levels and Breslow thickness [7, 8]. According to the survival advantage of female melanoma patients, men show significantly lower levels of ER $\beta$ in both melanoma and healthy tissues [7]. As widely known, melanoma presents a high percentage of genetic mutations. Although men accumulate a higher number of missense mutations (ratio Men to Women 1.85), their presence in metastatic melanoma results beneficial only for the overall survival in women, once again supporting the relevance of the functional pressure of the more efficient female immune system [9]. Conversely, the most common mutations involving B-Raf Proto-Oncogene (BRAF) and NRAS Proto-Oncogene (NRAS) seem to occur regardless of sex [10]. These "driver mutations" lead to constitutive activation of mutant signaling proteins that induce pathways supporting tumor onset [11]. Several efforts have been made to develop promising melanoma therapies, such as the combined target therapy against BRAF and Mitogen-Activated protein kinase (MEK) [12-14]. Regarding NRAS mutations, generally linked to poorer overall survival, targeted therapies based on
MEK inhibition as monotherapy or in combination with Mitogen Activated Protein Kinase (MAPK), Phosphoinositide 3-kinase (PI3K) or Cyclin Dependent Kinase (CDK) 4/6 inhibitors are under development although with disappointing results [15]. To overcome the specificity of each mutation, important results were obtained through different types of immunotherapy, like the immune checkpoint inhibitors (ICIs), really representing an improvement in anticancer strategies [16, 17]. Despite this progression in immuno- and target therapies, looking for the molecular mechanisms involved in different pathways of estrogen-dependent signaling in melanoma could help to understand the observed sex differences in disease severity, adverse events and outcomes, eventually opening an avenue towards a more personalized medicine. An important question to address concerns the existence of any relationship between estrogen receptor expression and oncogenic mutational status during disease progression. In this paper, we conducted a bioinformatics analysis in the Skin Cutaneous Melanoma (SKCM) patient database (The Cancer Genome Atlas, TCGA) to investigate both ERa and $\beta$ expressions in the total melanoma patient population and in BRAF and NRAS mutated subgroups, focusing on their modulation from primary to metastatic stage.

The unquestionable awareness of ER $\beta$ antitumor role led to the idea that any ligand capable of increasing ER $\beta$ expression or activity could be of great therapeutic utility, not only against melanoma. However, in the past years, many researchers experimented with different types of hormone agonists/antagonists or metabolites on melanoma, without reaching conclusive results [18]. Among them, the synthetic non-steroidal selective ER $\beta$ agonist LY500307 recently showed promising results, suppressing lung metastases in a mouse model of wild-type melanoma cell line, thanks to innate immunity increase [19]. Accordingly, LY500307 affects tumor growth in human glioblastoma and triple negative breast cancer [20, 21], both hormone sensitive tumors. Therefore, the exploitation of ER $\beta$ anticancer potential could represent a fruitful choice to counteract human melanoma, also in relation to melanoma mutational state.

Here, we unveil the possible molecular mechanism associated with ER $\beta$ signaling by using its agonist LY500307 on some human melanoma cell lines, characterized by different levels of ERs expression, genetic background and tumor progression. Interestingly, we demonstrated a selective effect of LY500307 in melanoma cell lines in terms of cell cycle blockage, apoptosis induction and partial EMT reversion, with particular reference to 
those expressing ER $\beta$ in NRAS-mutated genetic background.

\section{Methods}

\section{Cell lines and culture conditions}

Human melanoma cell lines used in the current study were previously described [22-24] (Table S1). Melanoma cell lines were periodically authenticated by standard short tandem repeat (STR)-based genotyping and the experimental analyses were always performed on controlled samples. Human fibroblast cell line (HF) used as normal control was kindly provided by the laboratory of Biotechnologies of the Experimental Medicine Department ("Sapienza", University of Rome, Italy) with patient written informed consent and ethical statement [25]. All the cells were tested for mycoplasma contamination with MycoAlert kit (LT07-418, Lonza) before use in experiments. Human melanoma cell lines were seeded in 6-well culture dishes in Dulbecco's modified Eagle's medium (DMEM, 61965-026) 2\% fetal bovine serum (FBS, 10270-106) $\mathrm{v} / \mathrm{v}$ and maintained in a humidified incubator $(5 \%$ $\mathrm{CO}_{2}, 37^{\circ} \mathrm{C}$ ). After three days, the cells were treated with LY500307 $(2,4,8 \mu \mathrm{M})$ (Erteberel, Cayman chemical, 22130), Pyrazolo[1,5-a] pyrimidines (PHTPP, 2.5 and $5 \mu \mathrm{M}$ ) (Selleckchem, 2662), Methyl-Piperidino-Pyrazole (MPP) dihydrochloride $(5 \mu \mathrm{M})$ (Tocris, 1991) or their vehicle (ethanol or DMSO) for different times $(5,24 \mathrm{~h})$ for cell cycle analyses and apoptosis assays. LY500307 was also used on normal human fibroblast and melanoma cell lines for proliferation/viability index evaluation (colorimetric assay XTT-based Roche Molecular Biochemicals, Mannheim, Germany).

\section{Flow Cytometry}

\section{Cell cycle analysis}

Melanoma treated cells were trypsinized and harvested in phosphate buffer saline (PBS), followed by fixation in ice-cold $70 \%$ ethanol overnight at $4^{\circ} \mathrm{C}$. About $3 \times 10^{5}$ cells were stained with a mixture of Propidium Iodide $(50 \mu \mathrm{g} / \mathrm{mL}$, Sigma-Aldrich, P4864) and RNAse A $(200 \mu \mathrm{g} / \mathrm{mL}$, Unimed, 501500) for 30 minutes at room temperature in the dark. The PI-stained cells were analyzed by flow cytometry (FCM) (GalliosTM, BD, San Jose, CA).

\section{Annexin $\mathrm{V}$ apoptosis assay}

Melanoma treated cells were trypsinized and washed twice in PBS1X. A proper number of cells $\left(3-5 \times 10^{5}\right)$ were resuspended in a mixture of Annexin binding buffer 1X (BD Biosciences, 556454), Annexin V647 (Life Technologies, A23204) and PI (Sigma
Aldrich, P4864) according to manufacturer's instructions. Cells were gently vortexed and incubated for 15 minutes at room temperature in the dark. The stained cells were analysed by using flow cytometry (GalliosTM, BD, San Jose, CA). This assay enables identification of both early (AV positive/PI negative), late (AV positive/PI positive) and necrotic (PI positive) cells.

\section{FCM analysis of extracellular E-cadherin}

Me1402/R melanoma cells were treated with LY500307 for $24 \mathrm{~h}$, trypsinized and washed twice in PBS1X. About $5 \times 10^{5}$ unfixed cells were stained on ice with primary antibody anti-E-cadherin recognizing the extracellular epitope and, subsequently, with the specific secondary antibody. Finally, cells were incubated with LIVE/DEAD ${ }^{\text {TM }}$ Viability/Cytotoxicity Kit (molecular probes by Life Technologies, L34962) and analyzed by CytoFLEX LX Flow Cytometer (UV-Violet-Blue-Yellow Green-Red (U-V-B-Y-R) Series, Beckman Coulter).

\section{Migration/Scratch assays}

Scratch wound assays were performed to measure migration and spreading capabilities of melanoma cells in vitro. The cells were seeded in 12-well culture plates and cultured in DMEM 10\% FBS v/v to nearly confluent cell monolayer. After that, a linear wound was generated in the cell monolayer (70-80\% of confluence) with a sterile p200 pipette tip. Any debris remaining in suspension were removed by washing cells once with PBS and then simple DMEM was replaced with DMEM $2 \%$ FBS with LY500307. Three representative images from the scratched area were captured (Bulldog Bio JuLi Smart fluorescent cell analyzer) to estimate the relative migration cells at different time points ( $\mathrm{t} 0$ and $24 \mathrm{~h}$ ). Data were analyzed quantitatively by using ImageJ (NIH) software, considering for each image the distances between one side of the scratch and the other.

\section{Western Blot and immunofluorescence analysis}

Western blotting was performed according to standard procedures. Total cells lysates were prepared by using NP40 cell lysis buffer, quantified by Bradford method and separated by the precast NuPAGE polyacrylamide gel system (Life Technologies Carlsbad, CA, USA). The expression levels were quantified using the AlphaView software (ProteinSimple San Josè, CA, USA).

Immunofluorescence analysis was performed according to standard procedures. Semi-confluent cells were grown and treated with LY500307 in 8-well chamber slides (Nalgene Nunc) and subsequently 
fixed in $4 \%(\mathrm{w} / \mathrm{v})$ paraformaldehyde (PFA, Sigma-Aldrich), permeabilized and saturated at room temperature. Incubations with primary and specific fluorophore-conjugated secondary antibodies (Alexa Fluor, Molecular Probes Eugene, OR, USA) were done in a humidified chamber at room temperature. Finally, cells were incubated with Hoechst 33342 to stain DNA and slides were mounted with ProLong without DAPI (Invitrogen, P36930). Cellular staining was analyzed by Olympus FV1000 laser-scanning confocal microscopy (Olympus, Tokyo, Japan).

\section{List of Utilized Antibodies}

All the antibodies listed below were used in accordance to the manufacturer's instructions: ERa (Santa Cruz, sc-787), ER $\beta$ (Abcam, \#455), Cyclin B1 (Santa Cruz, sc-7393), p21 (Santa Cruz Biotechnology, INC sc-817), Caspase-3 (Cell Signaling, \#9665), PARP (Cell Signaling, \#9532), Phalloidin (Alexa Fluor 488, \#A12379), Wee1 (Santa Cruz, \#5285), p-H2AX (Cell Signaling, \#9718), TWIST (Abcam, clone 2C1a \#ab50887), SLUG (Santa Cruz, sc-166476), E-cadherin (clone 36 BD \#610181), E-cadherin (HECD-1, Abcam, ab1416), $\beta$-actin (Sigma-Aldrich, Clone AC-15\#A5441) and Tubulin (Sigma-Aldrich clone B-5-1-2 T5168). As secondary antibodies, we used goat anti-mouse IgG (H+L) (\#115-035-166, Jackson ImmunoResearch) and goat anti-rabbit IgG (H+L) (\#65-6120, Invitrogen) for Western Blot analysis, anti-mouse AF488 (\#A11029) for IF analysis and AF647 (\#A21235) for cytometry (Alexa Fluor, Molecular Probes Eugene, OR, USA).

\section{Bioinformatics analysis}

The Illumina HiSeq RNA-seq dataset of 471 patients with Skin Cutaneous Melanoma (SKCM) was downloaded from the GDC Data Portal [26] by the TCGAbiolinks R package (ver 2.18.0) [27]. According to the subtype mutation, we extracted from the SKCM dataset the BRAFmut (patients with BRAF V600E mutation) and NRASmut (patients with NRAS Q61R mutation) subsets. Data preprocessing, normalization and quantile filtering was performed on each subset. By the TCGAbiolinks command embedding the edgeR $R$ package (ver 3.32.1) [28], we performed a differential expression analysis between Metastatic and Primary tumors in all subsets to investigate ERa and ER $\beta$ gene expression across conditions, applying a false discovery rate (FDR) threshold $<0.15$. The ratio between ERa and ER $\beta$ expression was also calculated in all the subgroups. Additional comparisons of ERa and ER $\beta$ gene expression as well as their ratio among patients with primary or metastatic tumor were performed by Generalized Liner Models analysis in $\mathrm{R}$ setting significance $\mathrm{p}<0.05$.

\section{Statistical Analysis}

Unless indicated otherwise, all data are presented as mean \pm standard deviation (SD) and results are representative of at least three independent experiments. Statistical analysis was performed using t-test, with $\mathrm{p}<0.05$ deemed statistically significant.

\section{Results}

\section{ESR 1 and ESR2 expression in SKCM patients}

Several efforts have been done in evaluating the expression levels of ERs in relation to the stage of melanoma progression with minor attention regarding the mutational state. Investigation of ERa and ER $\beta$ gene expression (ESR1 and ESR2, respectively) in SKCM patients from TCGA database (Fig. 1) revealed that no significant difference in ER1/2 gene levels was recorded between primary and metastatic patients in total SKCM, as well as BRAF or NRAS mutated subgroups. However, subjects with metastatic NRAS mutated tumors had significantly lower ERa $(\mathrm{p}<0.01)$ and $\operatorname{ER} \beta(\mathrm{p}<0.01)$ expressions compared to the total population of subjects with metastatic melanoma (Fig. 1). Regarding ERa, a decrease with borderline significance $(p=0.054)$ was recorded between patients with NRAS and BRAF primary tumors (Fig. 1). Since the balance between the two ERs expression might be more representative in defining estrogenic tissue-specific signaling, we analyzed the ERa/ER $\beta$ ratio between primary and metastatic melanoma both in the whole melanoma population and in the mutated subgroups. The significantly lower value observed in NRAS metastatic than in primary samples $(p<0.001)$ (Fig. 1) was suggestive of a more pronounced downregulation of ERa compared to ER $\beta$ in the advanced tumor stage.

\section{$E R \alpha$ and $E R \beta$ expression in melanoma cell lines}

The data obtained from TCGA analysis on melanoma patients led us to investigate ERa and ER $\beta$ expression levels in a panel of human melanoma cell lines stabilized from tumors at different stages of progression and genetic background (Table S1). By western blot assay, we detected high levels of ERa in the primary wild type melanoma lines Me1007 and Mel501 and in the BRAF mutated WM983A, it was weakly visible in Me1402/R, a mutated BRAF recurrence of primary melanoma, and barely detectable in NRAS-mutated metastatic Me665/1 and SK-Mel 30 cell lines (Fig. 2A). Interestingly, the highly invasive BRAF-mutated A375M showed a very high level of this protein, in agreement with ERa tumorigenic potential. Regarding ER $\beta$, we observed comparable levels in primary melanomas and in 
NRAS mutated metastatic cell lines, and lower, but well detectable levels in the BRAF mutated primary Me1402/R and metastatic A375M cell lines (Fig. 2B). Based on these results, we selected three melanoma cell lines for further studies, as representative of three different pathological conditions. We chose A375M and Me665/1, different in terms of genetic mutations but both extremely aggressive, as metastatic cell lines, together with Me1402/R melanoma cells, characterized by low expression of the oncomiR-221 and -222 and capability to melanin pigment synthesis [29], as a good model of primary melanoma capable to progress to a metastatic aggressive form.

\section{ESR1}

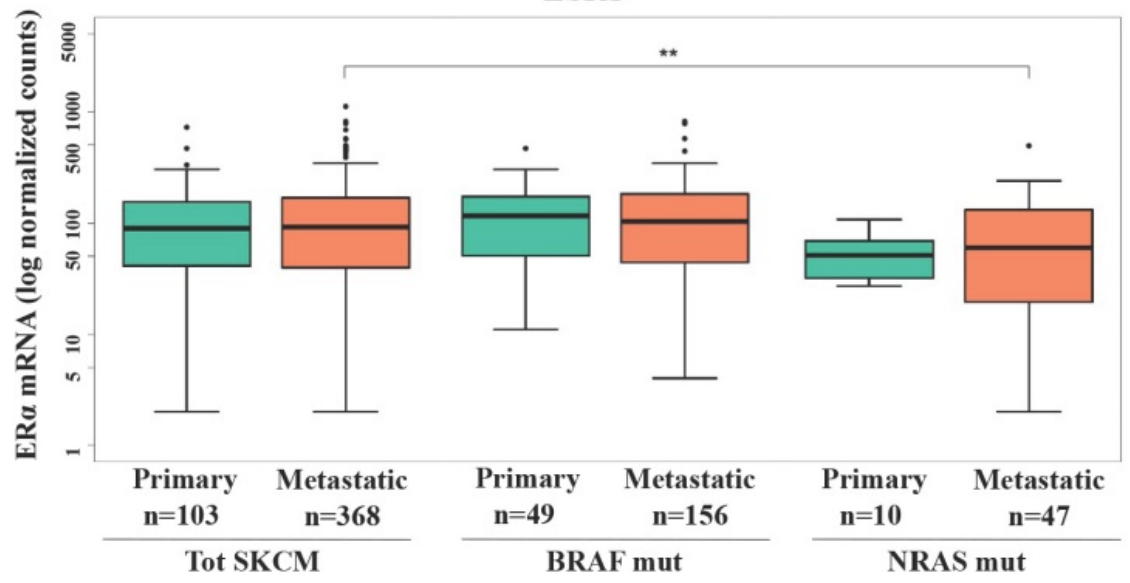

ESR2
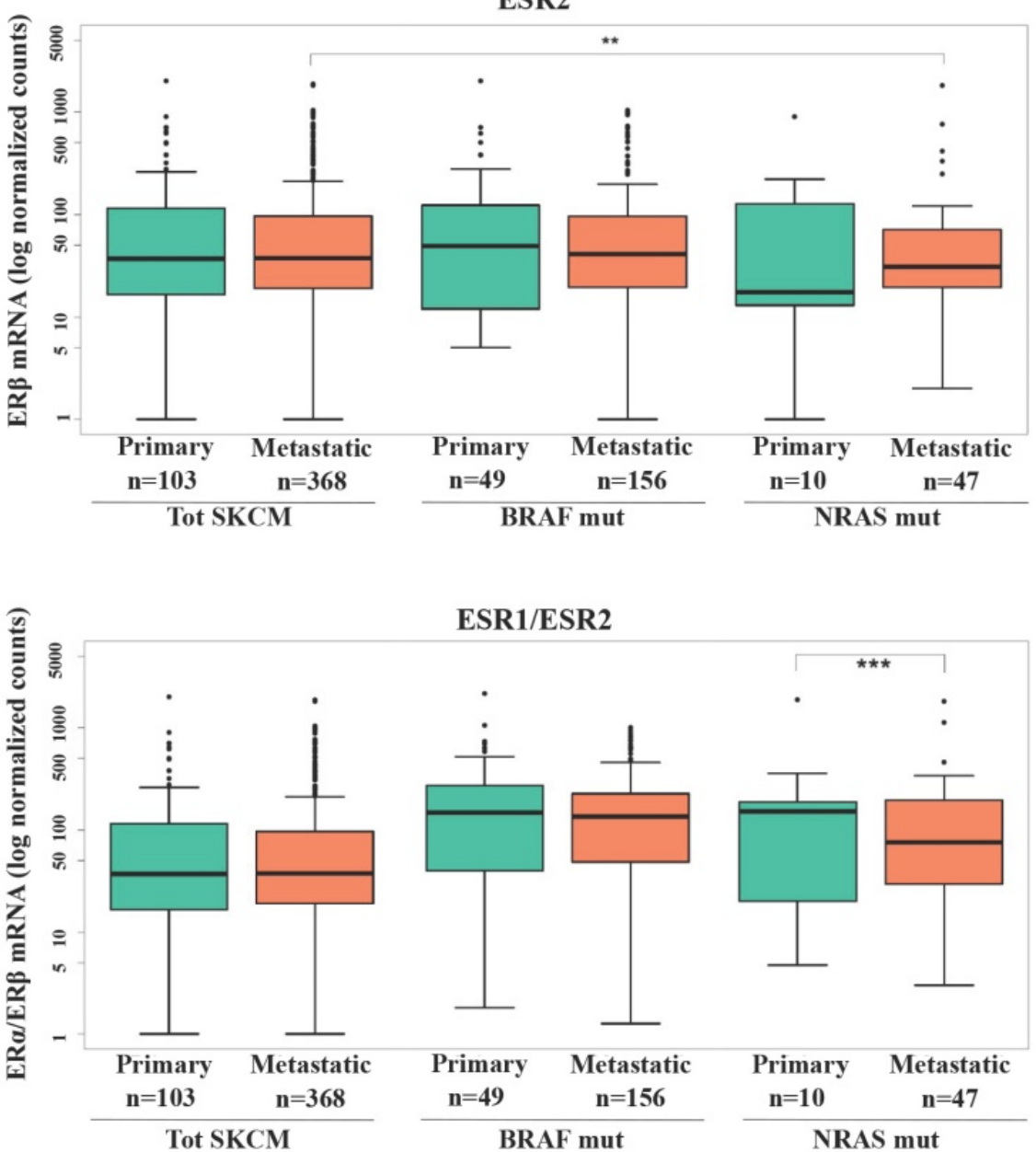

Figure 1. ERa and ER $\beta$ expression in SKCM patients. RNA-seq counts in logarithmic scale in total SKCM, BRAF and NRAS mutated patients with primary or metastatic tumors for ER $\alpha$ gene expression (ESR1), ER $\beta$ gene expression (ESR2) and ratio between ERa and ER $\beta$ gene expressions. Data are reported as interquartile (IQ) boxplots with inbox lines indicating median values and whiskers \pm 1.5 IQ range. Asterisks indicate the level of significance: $* * p<0.01 ; * * * p<0.001$. 


\section{Effects of Estrogen receptor $\beta$ selective agonist (LY500307) on melanoma cell lines proliferation and viability}

In order to investigate the potential ER $\beta$ antitumor role in melanoma upon receptor specific hormonal activation, we evaluated the effects of the specific ER $\beta$ agonist LY500307 on cell proliferation/viability of Me1402/R, A375M and Me665/1 melanoma cell lines (Fig. S1). Normal human primary fibroblast cell line (HF) was included in the viability assay, to exclude a non-specific toxic effect of LY500307 on cell proliferation. These cell lines were incubated with increasing doses of LY500307 $(2 \mu \mathrm{M}, 4 \mu \mathrm{M}, 8 \mu \mathrm{M})$ and cellular viability assessed at different time points. An initial reduction of the viability/proliferation index was observed in Me1402/R already after 24 hours of treatment followed by a strong increase in the following 48 and 72 hours. Concerning the metastatic cells, LY500307 treatment significantly reduced cell proliferation of Me665/1 as early as at 24 hours, whereas $48-72$ hours of treatment were necessary to detect a significant proliferative rate reduction in A375M. Notably, no viability decrease was observed in normal fibroblasts at all the tested doses and time points of treatment, suggesting LY500307 particular effectiveness on melanoma proliferation and viability, being nearly ineffective on normal cells.

\section{LY500307 affects melanoma cell cycle}

In view of the observed LY500307 effects on melanoma proliferation rate, we analyzed the cell cycle profile of the same cell lines in response to increasing concentration of LY500307 (from 2 to $8 \mu \mathrm{M})$. To this purpose, we cultured melanoma cells for three days in presence of $2 \%$ FBS DMEM thereafter adding LY500307 at the indicated concentrations. After 24 hours of treatment, we observed by flow cytometry analysis of PI-stained cells, a significant increase of Me1402/R cell percentage at the G2/M boundary phase compared to vehicle-treated controls at all LY500307 doses (Fig. 3A). More significant blockage at $\mathrm{G} 2 / \mathrm{M}$ boundary phase was detected in Me665/1, especially at the higher concentrations (Fig. 3B), whereas A375M cell cycle was barely affected only at highest LY500307 dose (Fig. 3C). Similar results were obtained treating Me665/1 in medium with $2 \%$ charcoal serum, in order to provide hormone-free cell culture conditions (Fig. S2A). The time course of LY500307 treatment confirmed the greater sensitivity of Me665/1 cells that began to accumulate in G2/M boundary phase already after 5 hours. This effect was detectable to a lesser extent in Me1402/R and not at all in A375M cells (data not shown). Similar results were obtained in the SK-Mel 30, another NRAS-mutated metastatic melanoma cell line (Fig. S3A).
$\mathbf{A}$

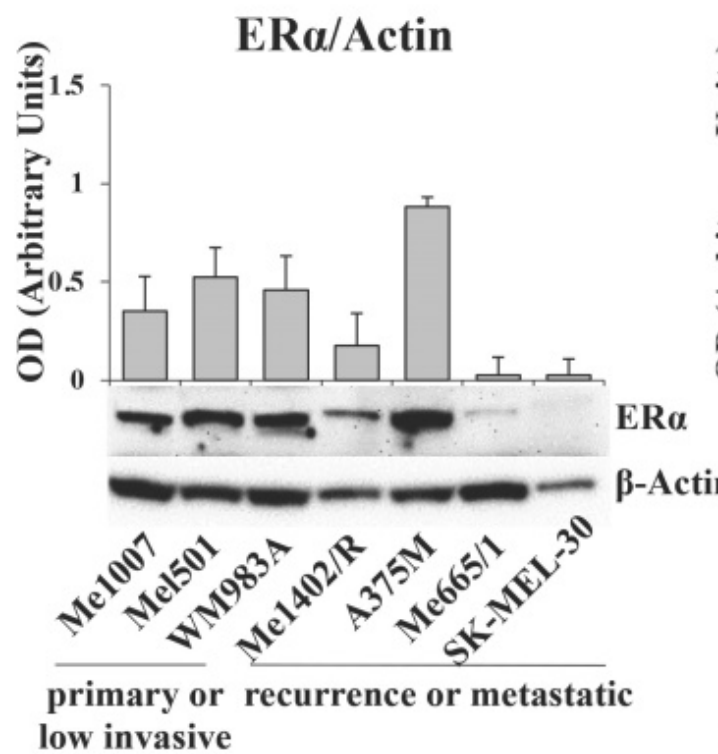

B

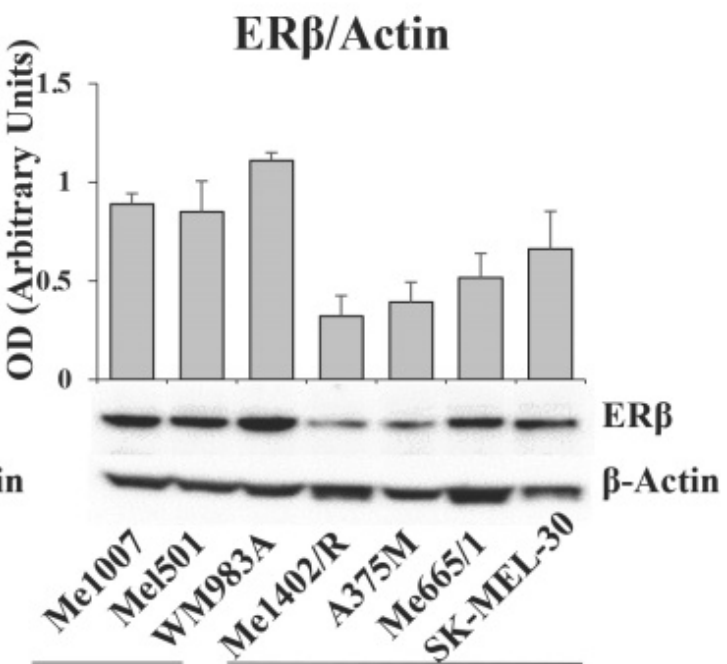

primary or recurrence or metastatic low invasive

Figure 2. ER $\alpha$ and ER $\beta$ expression levels in different tumor staged melanoma cell lines. Western blot analysis of (A) ER a and (B) ER $\beta$ in primary (Me1007, Mel501, WM983A), recurrence (Me1402/R) and metastatic (A375M, Me665/1, SK-MEL-30) melanoma cell lines, and corresponding relative densitometric quantification. $\beta$-Actin was utilized as internal loading control. Data are expressed as the mean \pm SD of three independent analyses. 
Since LY500307 was described as a potent and selective ER $\beta$ agonist [20], to confirm our data we performed some competitive experiments with the highly specific antagonist of ER $\beta$, PHTPP [30] on Me665/1, as best responsive cell line. Me665/1 cells were treated with either LY500307 $(4 \mu \mathrm{M}$ and $8 \mu \mathrm{M})$ or PHTPP $(5 \mu \mathrm{M})$, alone or in combination, for 24 hours. Results indicated that the co-administration of LY500307 and PHTPP significantly reduced the effect obtained on cell cycle with LY500307 alone, confirming its selectivity for the ER $\beta$ isoform (Fig. 3D). The PHTPP inhibitory effect was stronger at 4 $\mu \mathrm{M}$ LY500307, possibly because at $8 \mu \mathrm{M}$ dose the G2/M block was too advanced.

Despite the low ERa level in Me665/1, we wondered if the combined treatment of Me665/1 with LY500307 and the selective ERa antagonist MPP (5 $\mu \mathrm{M})$ [31] could improve the effect of LY500307 treatment. Interestingly, the effect of the ER $\beta$ agonist on cell cycle was improved by blocking ERa, whereas MPP treatment was ineffective when used alone (Fig. 3E).

Cell cycle arrest is characterized by the modulation of several cell cycle regulators [32]. Among them, relevant roles are played by $\mathrm{p} 21$, a well-known inhibitor of cyclin dependent kinases, and Cyclin B1, whose expression and cellular localization are finely regulated during the cell cycle phases [33]. Western blot analysis of p21 expression showed a direct correlation between p21 up regulation and drug amount in all melanoma cell lines treated with LY500307. This modulation was more evident in Me665/1 (Fig. 4B) and SK-Mel 30 cell lines (Fig. S3B) characterized by NRAS mutation, and less clear in Me1402/R (Fig. 4A) and A375M (Fig. 4C) BRAF-mutated cells. These results are consistent with the variability of responses to LY500307 treatment in terms of cell cycle progression in the selected cell lines. Conversely, Cyclin B1 expression remained constant in all the analyzed melanoma cell lines except at $8 \mu \mathrm{M}$ dose, where it suddenly increased (Fig. 4A, B, C). Regarding Cyclin B1, similar results were obtained in SK-Mel 30 cell line (Fig. S3B) and in Me665/1 treated in culture medium supplemented with charcoal (Fig. S2B). Since cyclin B1 levels oscillate over the course of the cell cycle, being degraded on metaphase-anaphase transition [34], its constant expression observed at 2 and $4 \mu \mathrm{M}$ and its up-modulation at $8 \mu \mathrm{M}$ of LY500307 is in line with the G2/M phase arrest.
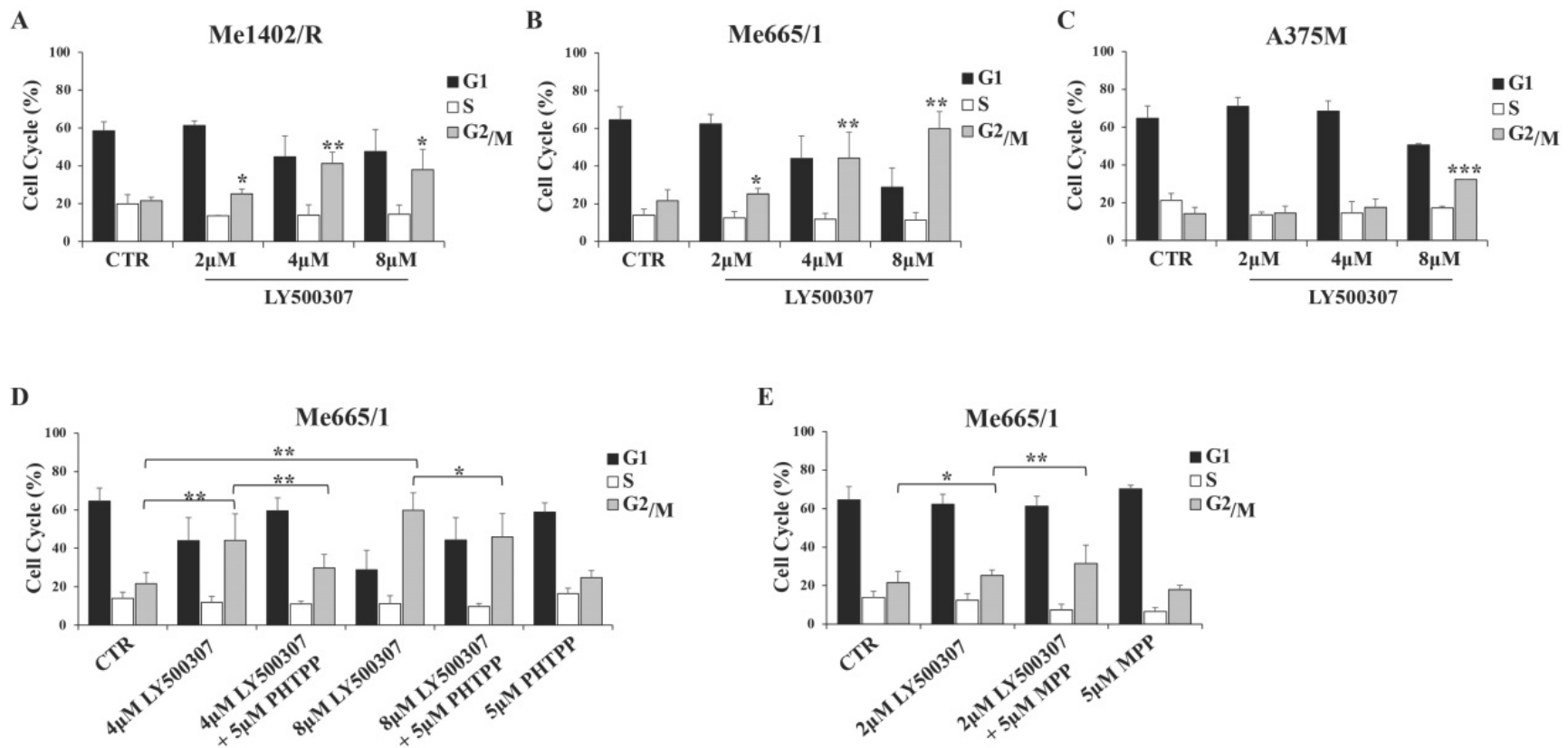

Figure 3. LY500307 and its combined treatment with ERß/a antagonists (PHTPP, MPP) on melanoma cell cycle. Cell cycle analysis of control (CTR) vs cells

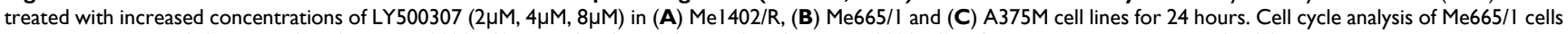
treated with either (D) PHTPP $(5 \mu M)$ and LY500307 $(4 \mu$ M or $8 \mu M)$ or (E) MPP $(5 \mu M)$ and LY500307 $(2 \mu M)$ alone or in combination for 24 hours respect to the untreated control (CTR). Data are represented as mean \pm SD of three independent experiments. Asterisks indicate the level of significance: ${ }^{*} p<0.05 ; * * p<0.01 ; * * * p<0.001$ compared with the corresponding CTR or LY500307 treated cells. 
A

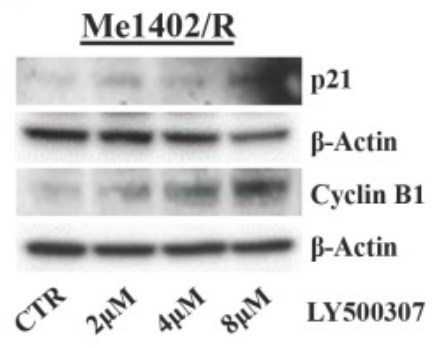

B

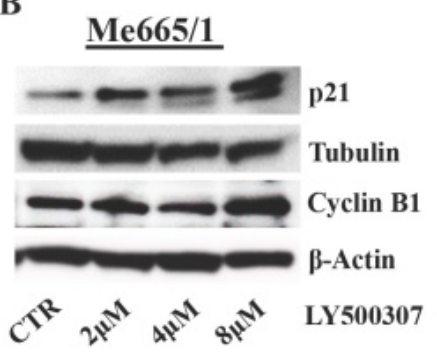

C

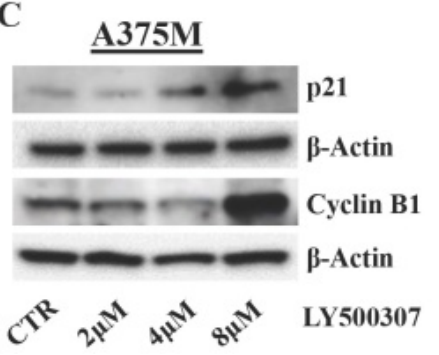

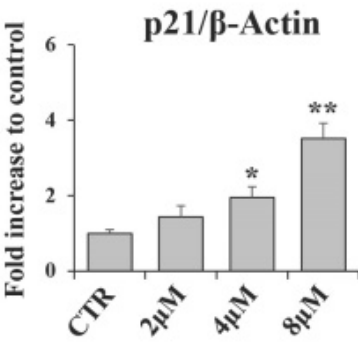
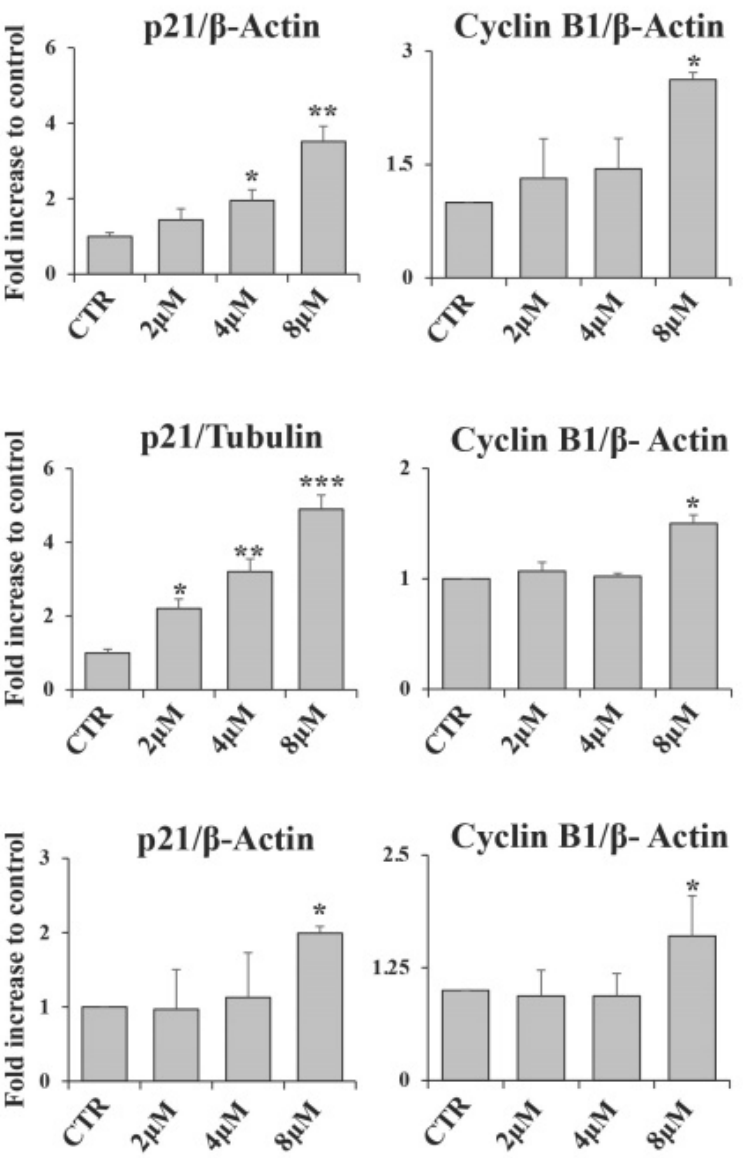

Figure 4. Evaluation of cell cycle regulators. Representative Western Blots of $\mathrm{p} 21$ and cyclin $\mathrm{B} 1$ expression levels in Me1402/R (A), Me665/1 (B) and A375M (C) cells treated with increased concentrations of LY500307 $(2 \mu \mathrm{M}, 4 \mu \mathrm{M}, 8 \mu \mathrm{M})$ for 24 hours. $\beta$-Actin and Tubulin were utilized as internal loading control. Densitometric quantifications shown as fold increase are represented as mean \pm SD of three independent experiments. Asterisks indicate the level of significance: ${ }^{*} \mathrm{P}<0.05 ; * * \mathrm{P}<0.01 ; * * * \mathrm{P}<0.001$ compared with the control cells (CTR).

\section{LY500307 drives melanoma cells towards mitotic catastrophe events}

The observed cell cycle arrest at G2/M boundary upon LY500307 treatment led us to investigate the nuclear morphology of melanoma cell lines with particular attention to the presence of macroscopic anomalies in the mitotic figures. We performed immunofluorescence analyses focusing on DNA and mitotic spindle structure, based on Hoechst and tubulin staining, respectively. Confocal analysis showed that LY500307 treatment determined anomalous mitosis, starting from misaligned metaphasic plaques, up to the formation of small nuclear bodies containing DNA fragments, similar to a mitotic catastrophe event. In agreement with the effect of LY500307 on the cell cycle, we observed abnormal mitosis both in Me1402/R (Fig. 5A) and in Me665/1 (Fig. 5B) after 12 and 5 hours of treatment, respectively. As expected, only after 24 hours of treatment with the highest dose of LY500307, few defective mitoses were detectable in the less responsive A375M cells (Fig. 5C).
As cell cycle arrest consists of a series of events often activated by cells in response to DNA damage, we focused our attention on Wee1-like protein kinase (Wee1) and phospho-H2A histone family member $\mathrm{X}$ (p-H2AX), two key factors involved in this process. Wee1 is a mitotic inhibitor [35] and H2AX is a histone variant undergoing a rapid phosphorylation in case of DNA injury [36]. Western blot analysis showed a significant inverse correlation between Wee1 expression and LY500307 treatment in both Me1402/R and Me665/1, but not in A375M (Fig. 6A, $\mathrm{B}, \mathrm{C})$. On the other hand, we found a sharp increase of p-H2AX starting from $4 \mu \mathrm{M}$ of LY500307 in Me665/1 (Fig. 6B) and, to a lesser extent, in $\mathrm{Me1402/R}$ and A375M cell lines (Fig. 6A, C).

The opposite expression profiles of Wee1 and p-H2AX suggest that LY500307 might induce the defective cells to bypass DNA damage repair processes and to enter prematurely in mitosis, resulting in improper chromosome segregation eventually leading to mitotic catastrophe and apoptosis. 


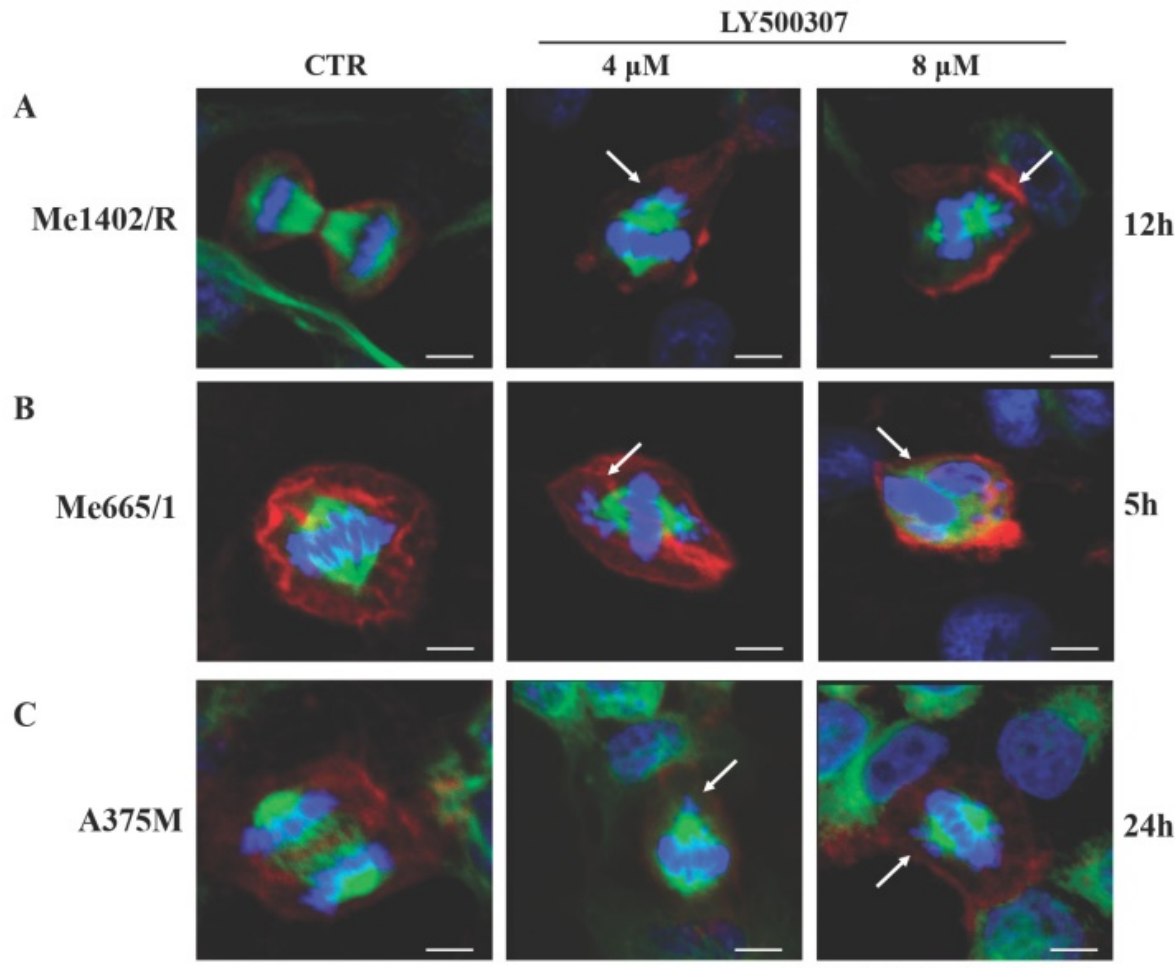

(DNA, Tubulin, Phalloidin)

Figure 5. LY500307 treatment induces alteration in mitotic figures. Confocal microscopy visualization of cellular and nuclear morphology of (A) Mel402/R, (B) Me665/1 and (C) A375M melanoma cell lines after 12, 5 and 24 hours of LY500307 treatment, respectively. Cells were stained with Phalloidin (Alexa Fluor 647-red) and a-tubulin (Alexa Fluor488-green) for visualization of actin and tubulin filaments, respectively. Nuclei were counter-stained with Hoechst 33342 (blue). Scale bar: $10 \mu \mathrm{MM}$

A

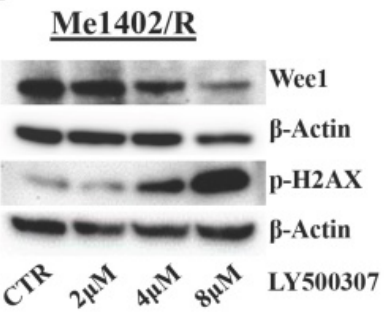

B

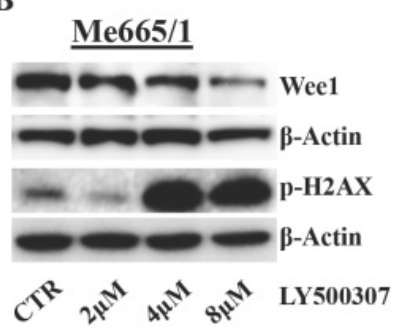

C

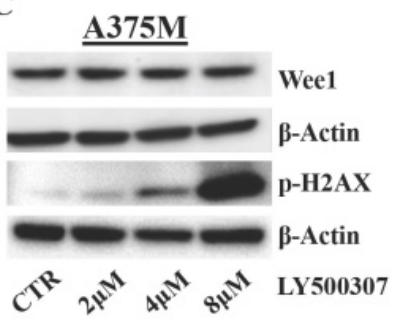

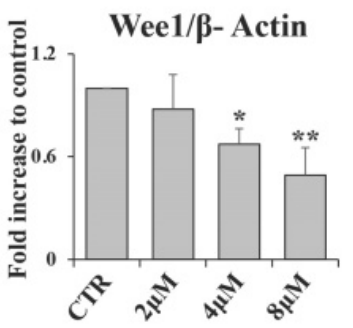
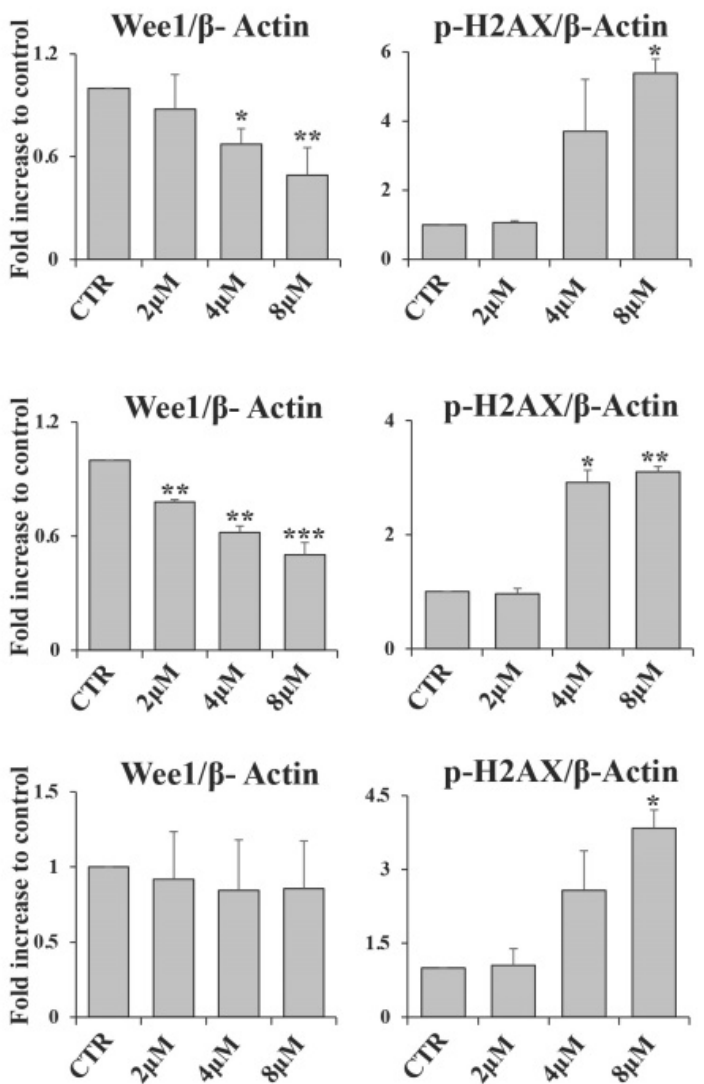

Figure 6. LY500307 treatment modulates the expression levels of DNA damage related proteins. Representative Western Blot analysis of Weel and p-H2AX

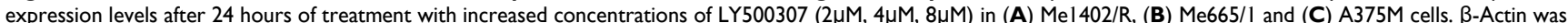
utilized as internal loading control. Densitometric quantifications, shown as fold increase, are represented as mean \pm SD of three independent experiments. Asterisks indicate the level of significance: $* p<0.05, * * p<0.01, * * * p<0.001$ compared with the control cells (CTR). 


\section{LY500307 induces apoptosis in melanoma cells}

In order to evaluate whether melanoma cells blocked in G2/M undergo apoptosis, we analyzed by western blot some specific apoptotic markers, such as caspase 3 and Poly (ADP-Ribose) Polymerase (PARP), and, in parallel, the amount of Annexin V/PI positive cells by Flow Cytometry (FCM). After 24 hours of LY500307 treatment, Me1402/R showed a dose dependent cleavage of both caspase 3 and PARP, statistically significant at $4 \mu \mathrm{M}$ and $8 \mu \mathrm{M}$ of LY500307 (Fig. 7A). Accordingly, FCM analysis showed a progressive increase of late-apoptotic cells (Annexin $\mathrm{V} /$ PI-double-positive cells), directly proportional to the treatment dose (Fig. 7B).

Me665/1 cells showed activation of both caspase 3 and cleaved PARP (Fig. 7C). FCM analysis of PI/ Annexin V, showed a sharp increase of both earlyand late-apoptotic cells (Annexin $\mathrm{V}^{+} / \mathrm{PI}$ and Annexin $\mathrm{V}^{+} / \mathrm{PI}^{+}$-positive cells) proportional to LY500307 concentration (Fig. 7B).

Conversely and according to cell cycle analysis, A375M cells revealed a slight activation of PARP and caspase 3 only at $8 \mu \mathrm{M}$ of LY500307 (Fig. 7D) and no increase of PI/Annexin V positive cells (data not shown).

\section{LY500307 counteracts melanoma cell motility and modulates the expression of epithelial- mesenchymal transition transcription factors (EMT-TFs)}

Migration and invasion capabilities of tumor cells play an important role in spreading and metastasization [37] and ER $\beta$ activation has often been involved in epithelial versus mesenchymal transition inhibition, as in breast and prostate cancers $[38,39]$. Therefore, by in vitro scratch assays performed in low serum condition, we evaluated the effect of LY500307 on the migration ability of melanoma cell lines (Fig. 8A, B, C). Interestingly, we observed a significant dose-dependent reduction of cell migration with respect to the corresponding untreated cells, irrespective of the closure or not of wound area in the controls. Particularly, for the less aggressive Me1402/R cells, it is conceivable that low serum culture condition per se influenced the rate of cell migration (Fig. 8A). Since EMT underlies the increased aggressiveness of advanced tumors, that lose the epithelial characteristics acquiring the mesenchymal ones, and based on the reduced dissemination of LY500307-treated cells, we investigated whether LY500307 could modulate some key transcription factors (i.e. SLUG and TWIST) as well as the adhesion molecule E-cadherin, favoring a mesenchymal-epithelial transition (MET) [40]. Indeed, although at different levels, western blot analysis demonstrated a general dose-dependent decrease of SLUG and TWIST expression in melanoma cells (Fig. 9A, B, C).

Interestingly, in Me1402/R the decreased expression of these EMT-TFs was associated with a gradual increase of E-cadherin expression, which became significant at the highest concentration of LY500307 (Fig. 9D). It is important to evidence that this increase was associated with exposure of E-cadherin on cell membrane surface, as demonstrated by flow cytometric analysis of viable cells stained with antibody recognizing the extracellular domain of E-cadherin (Fig. 9E). This last result is suggestive of epithelial-mesenchymal reversion onset in Me1402/R cells, an event conceivable for a local relapse-derived melanoma cell line with most of the characteristics of the primary tumor.

\section{Discussion}

Epidemiological data show a significant gender difference in melanoma incidence, partly based on the pivotal role played by sex hormones [18]. In this respect, ER $\beta$ displays a suppressive activity [41] and low expression of its mRNA correlates with poorer relapse-free survival of melanoma patients [42]. Regarding ERa, an interesting association between its gene polymorphisms and some clinical parameters has been shown [43]. To our knowledge, no information has been reported so far on a possible correlation between ER1/2 expression and mutational status of melanoma. In this regard, our analysis, conducted on the TCGA database, did not show a significant difference in ER expression associated with melanoma progression. Nevertheless, significantly lower levels of both ERa and ER $\beta$ were found in the subgroup of metastatic NRAS mutated samples compared to the total metastatic population. Of note, the lower $E R a / \beta$ ratio observed in metastatic versus primary NRAS mutated melanomas suggests a greater decrease of ERa than ER $\beta$ expression. In line with that, ER protein levels evaluated in different melanoma cell lines, showed the lowest ERa amount in NRAS metastatic cells Me665/1 and SK-Mel 30, whereas ER $\beta$, albeit lower respect to primary tumor, remained well detectable. Conversely to our TCGA analysis, the BRAF-mutated A375M showed an elevated expression of ERa, thus representing a model of metastatic melanoma, opposite to the NRAS ones in our study. As reported before, Zhao and colleagues, showed the capability of the synthetic non-steroidal selective ER $\beta$ agonist LY500307 to reduce melanoma lung metastasis in a murine melanoma in vivo model, by innate immunity upregulation in the metastatic 
niche [19]. Although of interest, this study was focused on B16F10 mouse metastatic melanoma cell line, wild-type respect to either BRAF or NRAS mutations, and indicated the tumor microenvironment as key mediator of LY500307 effects, without evidence of any cell-dependent pathway activation. Therefore, in order to translate the LY500307 function to human disease, we focused our study on some representative human melanoma cell lines.
A

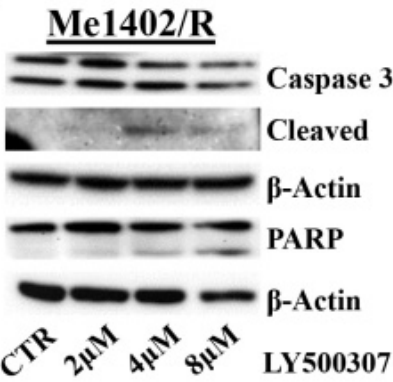

Caspase 3

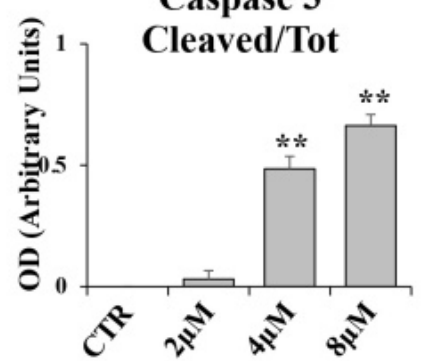

PARP

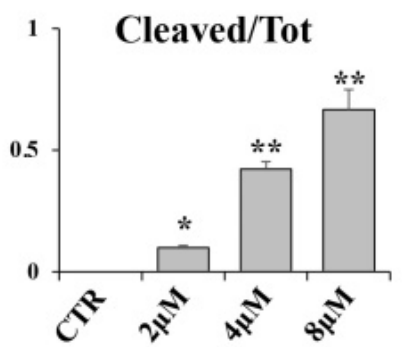

B

LY500307

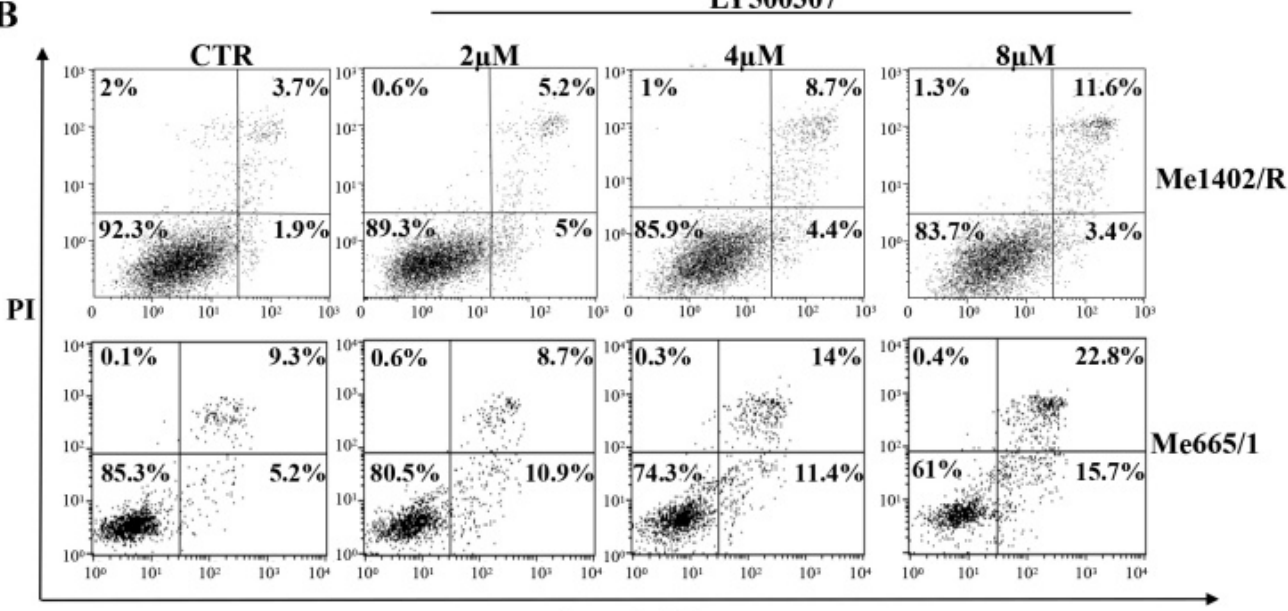

Annexin V

C

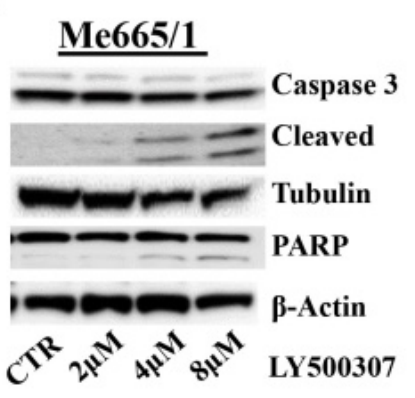

Caspase 3

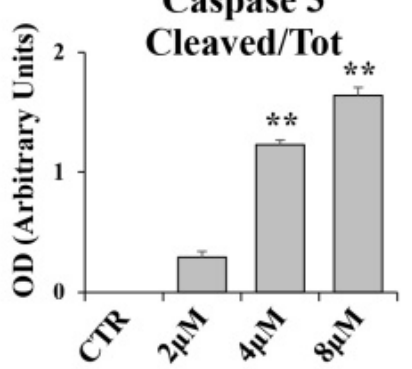

PARP

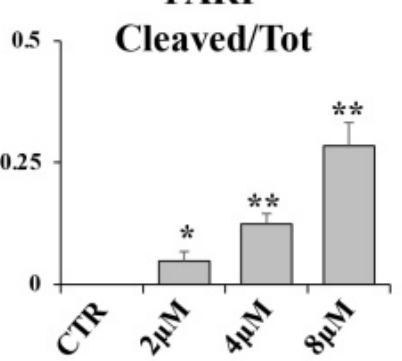

D
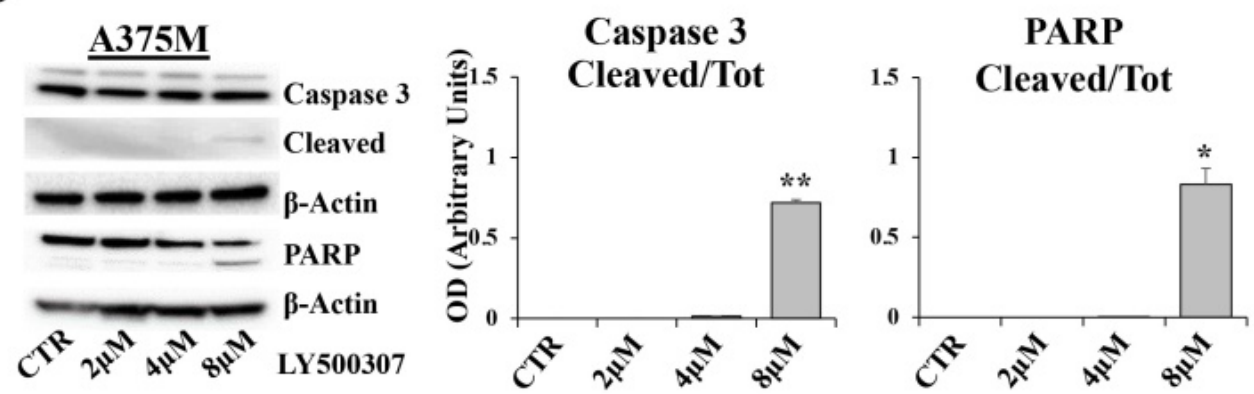

Figure 7. LY500307 induces apoptosis in Me1402R cell line. Representative Western Blots illustrate expression levels of Caspase 3, PARP and their cleaved forms in

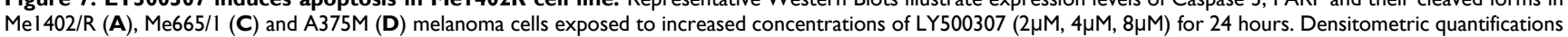


of Cleaved/Total Caspase-3 and PARP ratios are represented as mean \pm SD of three independent experiments. Quantification of apoptotic cell fractions of Me1402R and

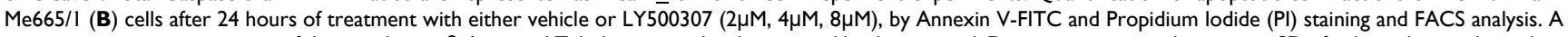
representative experiment out of three is shown. $\beta$-Actin and Tubulin were utilized as internal loading control. Data are represented as mean \pm SD of at least three independent experiments. Asterisks indicate the level of significance: ${ }^{*} p<0.05, * * p<0.01, * * * p<0.001$ compared with the control cells (CTR).
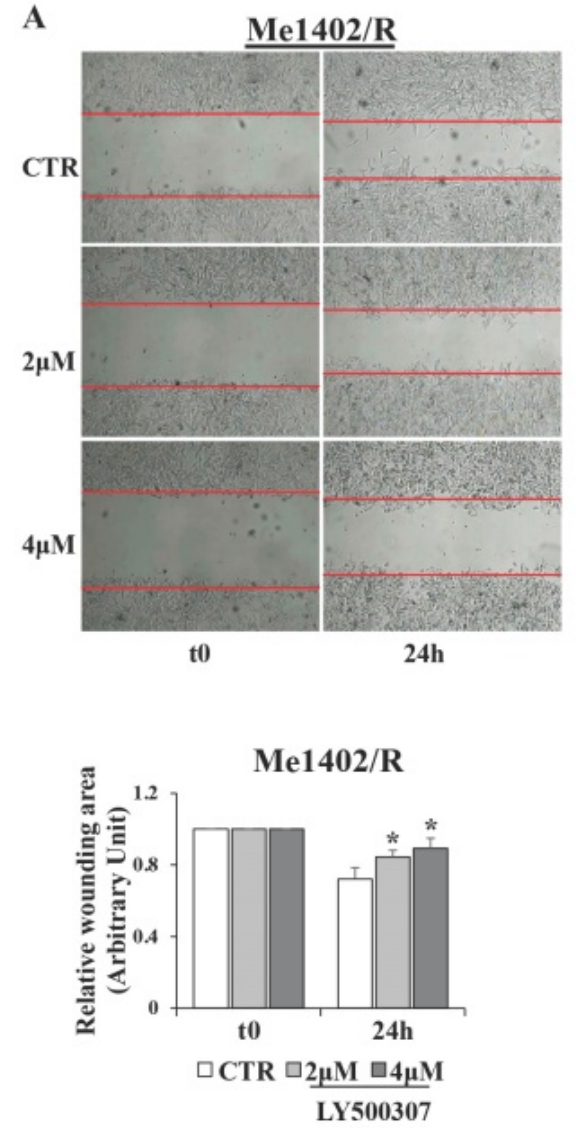
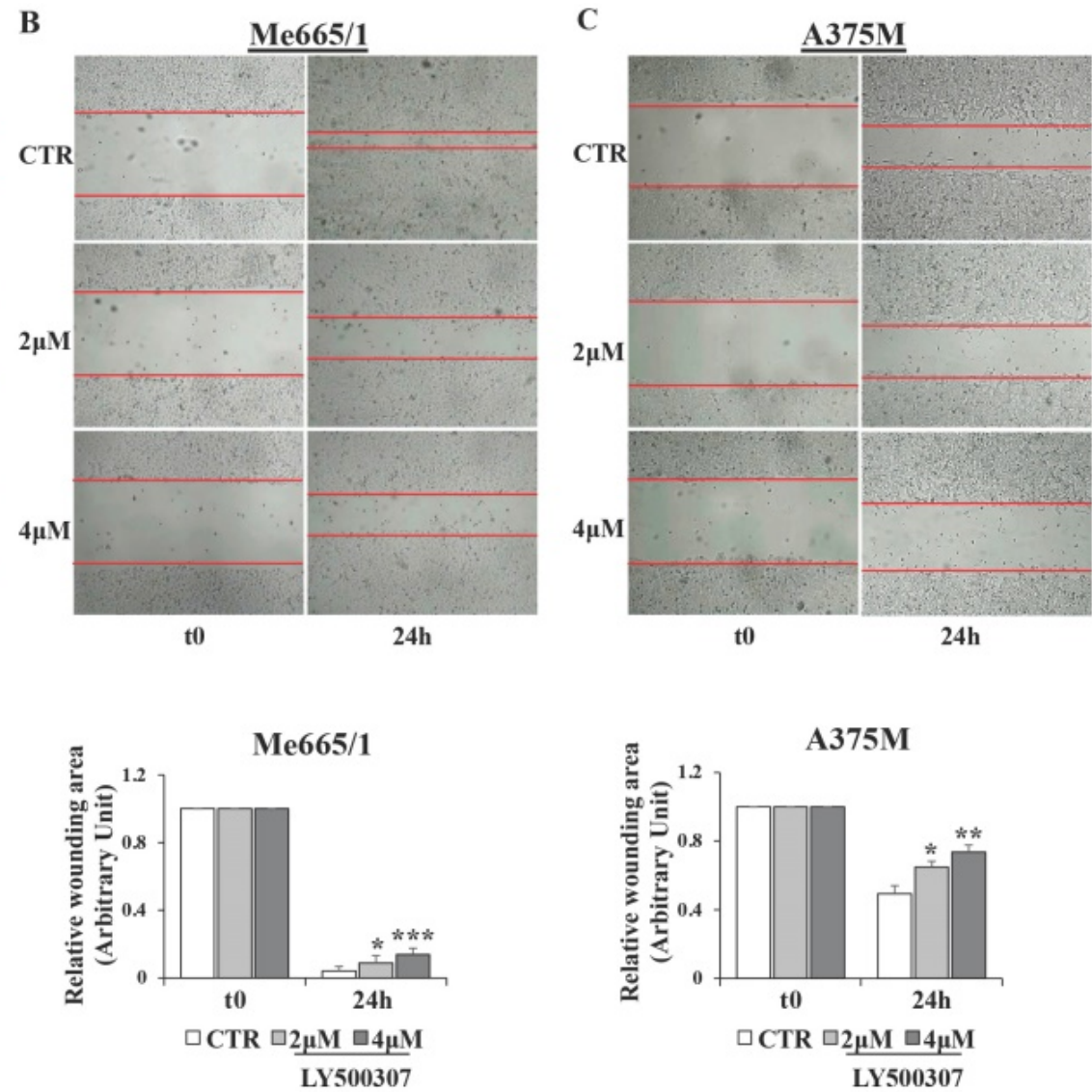

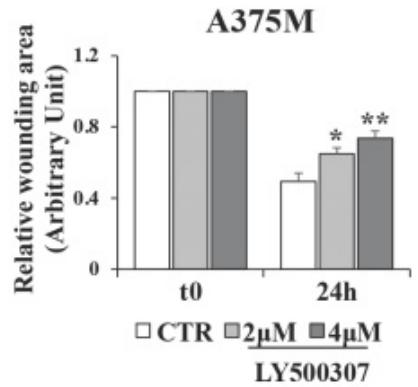

Figure 8. Analysis of cell migration by scratch assay in melanoma cell lines after LY500307 treatment. Representative time-lapse microscopy images (upper panel)

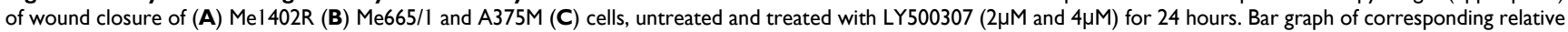
wounding area (lower panel). Results represent the mean \pm SD of at least four measurements of each wounded area, obtained in three independent experiments. Asterisks indicate the level of significance: $* p<0.05$, ** $p<0.01$, *** $p<0.001$ compared with the control cells (CTR).

Here, we demonstrated the ability of this compound to affect melanoma cell proliferation with minimal toxicity on normal cells (Fig. S1), inducing cell cycle arrest in G2/M phase in agreement with the absolute amount of ER $\beta$ or the relative expression of $E R a$ and ER $\beta$ in each melanoma cell line (Fig. 3A, S2). Interestingly, the ER $\beta$ antagonist PHTPP (Fig. 3D) was able to attenuate LY500307 effect and, possibly more important, the ERa antagonist MPP increased the cell sensitivity to a lower dose of LY500307 (Fig. 3E). Suppression of proliferation through blockade of cell cycle progression has also been observed in other two sex hormone-related cancers, as glioblastoma [20] and triple negative breast cancer (TNBC) [21].

Interestingly, this G2/M cell cycle block was associated with reduced Wee1 kinase expression and induction of histone H2AX phosphorylation (Fig. 6). Hence, we can assume that this blockage is a consequence of deficiency in some cell cycle checkpoints leading cells to enter mitosis before genomic instability resolution and in turn undergoing mitotic catastrophe. Interestingly and differently to what observed in the B16-based mice model [19], LY500307 was able to induce a cell-autonomous apoptotic program in these in vitro treated human melanoma cell lines. Therefore, LY500307 appears to exert a double activity against cancer, decreasing tumor growth and increasing the availability of tumor specific antigens to be recognized as non-self by the immune system, as shown by reduced proliferation and apoptosis onset. To note, a recent study in murine Triple-Negative Breast Cancer (TNBC) and colorectal cancer cells demonstrated that combined treatment of ER $\beta$ agonist and PD-1 antibody, reduced Myeloid-Derived Suppressor Cells (MDSC) tumor infiltration and enhanced its response to ICB therapy [44]. 


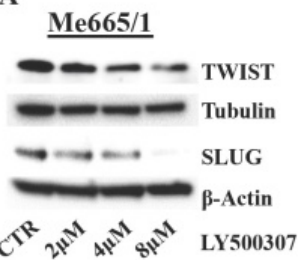

B

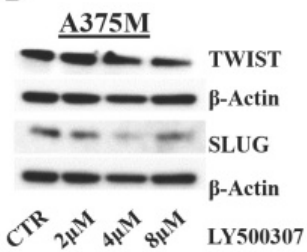

C

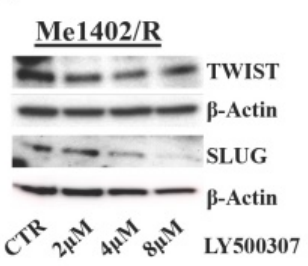

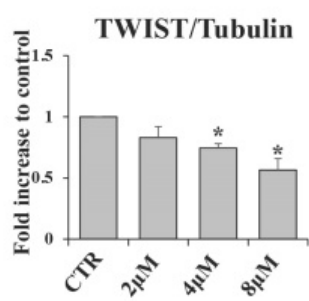
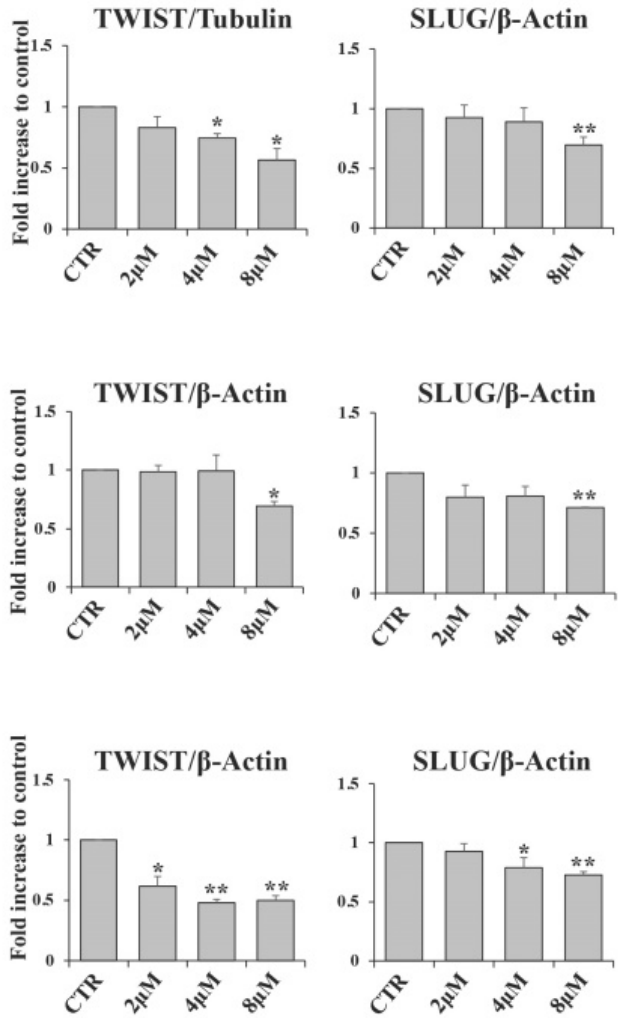

D
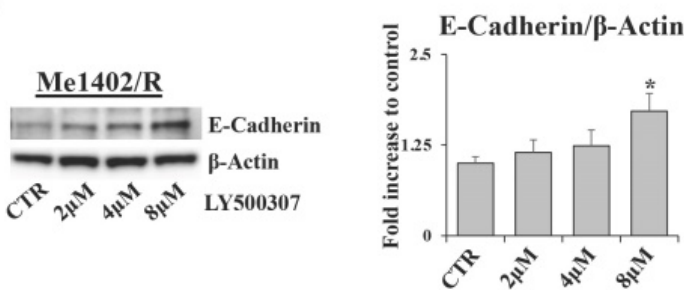

$\mathbf{E}$
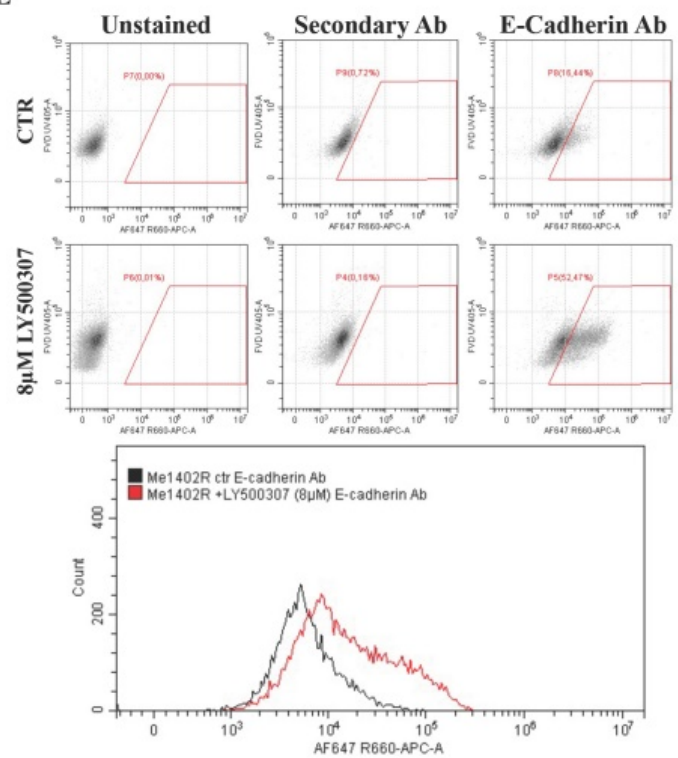

Figure 9. Effect of LY500307 treatment on EMT-TFs. Representative WB analysis of TWIST and SLUG in Me665/1 (A), A375M (B) and Me1402/R (C) after 24h of

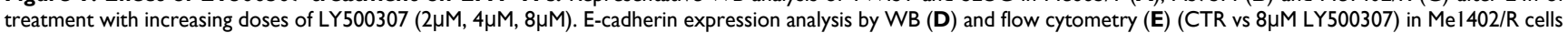
treated for 24 hours with LY500307. A representative FCM experiment out of three is shown. $\beta$-Actin or Tubulin were utilized as internal loading control. Densitometric quantifications shown as fold increase are represented as mean \pm SD of three independent experiments. Asterisks indicate the level of significance: $* p<0.05$, $* * p<0.01$ compared with the control cells (CTR).

According to the supposed ER $\beta$ antimetastatic activity, LY500307 resulted able also to reduce cell migration (Fig. 8) and down-modulate some of the transcription factors involved in the epithelialmesenchymal process (Fig. 9). Interestingly, in the less aggressive Me1402/R cells, E-cadherin was increased and correctly localized on the membrane surface, suggestive of a more complete mesenchymalepithelial reversion (Fig. 9D, E), easier to obtain in vitro in this melanoma cell line, with still some molecular traits typical of primary stage. It is worth noting that ERa did not counteract ER $\beta$ functional activity on the invasion capacity suggesting that both cell migration and MET could be modulated by ER $\beta$ agonist via molecular pathways not influenced by ERa signaling. Accordingly, reduced cell migration associated with E-cadherin upregulation by ER $\beta$ expression and further increased by LY500307 treatment was recently demonstrated in TNBC [45].

Overall, our work demonstrated the oncosuppressive effect of ER $\beta$ activation on some representative human melanoma cell lines, characterized by different levels of ERs expression, genetic background and tumor progression level.

Further in vivo studies will shed light on the possible role of ER $\beta$ in counteracting melanoma metastasization, considering the possible influences of the tumor microenvironment besides the direct actions of LY500307 here reported on melanoma cells. Indeed, although impressive therapeutic results have been achieved for the treatment of melanoma over the past decade, effective clinical results are still lacking for NRAS-positive melanoma patients. This is partly due to the low number of NRAS positive patients, which also limited ER expression studies, especially in view of a sex- and age-stratified investigation. As suggested by data obtained with the ER $\beta$ agonist LY500307, we could consider the option of ER $\beta$ as a candidate for alternative and combined therapeutic strategies. A definite comprehension of the molecular pathways underlying the ER $\beta$ effectiveness in mutated NRAS cells would dissect the direct and indirect mechanisms, exploiting the ER $\beta$ antitumor potential possibly associated with the different mutations underlying melanoma.

\section{Conclusions}

Our results demonstrate that the selective ER $\beta$ agonist LY500307 differently affects melanoma cell proliferation, related to stage, genetic background and 
ERs expression. These data confirm the ER $\beta$ ability to counteract melanoma onset and progression, suggesting the importance of determining both the patient's genetic profile and ERs expression in tumor tissues for selecting the most effective and personalized therapeutic approach.

\section{Abbreviations}

BRAF: B-Raf Proto-Oncogene; CDK4/6: Cyclin Dependente Kinase 4/6; EMT: EpithelialMesenchymal Transition; EMT-TFs: EpithelialMesenchymal Transition Transcription factors; ERs: Estrogen Receptors; FCM: Flow cytometry; HF: Human Fibroblast; ICIs: Immune Checkpoint Inhibitors; MAPK: Mitogen-Activated Protein Kinase; MDSC: Myeloid-Derived Suppressor Cells; MEK: Mitogen-Activated Protein Kinase; MPP: MethylPiperidino-Pyrazole; NRAS: NRAS Proto-Oncogene; p-H2AX: phospo-H2A histone family member X; PARP: Poly (ADP-Ribose) Polymerase; PHTPP: Pyrazolo[1,5-a] pyrimidines; PI3K: Phosphoinositide 3-Kinase; SKCM: Skin cutaneous melanoma; TCGA: The cancer genome atlas; TNBC: Triple-Negative Breast Cancer; Wee1: Wee1-Like Protein Kinase.

\section{Supplementary Material}

Supplementary figures.

https://www.jcancer.org/v13p1573s1.pdf

\section{Acknowledgments}

We thank patients and investigators who participated in TCGA for providing data. We thank Antonella Torrice and Daniela Farinelli for the administrative support.

\section{Funding}

This work was partially supported by the Italian Association for Cancer Research (AIRC IG18815 to AC) and by the internal funds of Istituto Superiore di Sanità.

\section{Author Contributions}

$\mathrm{RP}$ designed the study; GP, RP, MB and PM performed the experiments and analyzed the data, $\mathrm{RP}$ and GP wrote the manuscript; ST performed data bioinformatics analysis, VT performed flow cytometry analysis, AC and GM participated in study coordination and critically reviewed this manuscript. All authors read and approved the final manuscript.

\section{Competing Interests}

The authors have declared that no competing interest exists.

\section{References}

1. Italian Association of Medical Oncology (AIOM): The Numbers of Cancer in Italy 2020. https://www.aiom.it/i-numeri-del-cancro-in-italia/.

2. Taneja V. Sex Hormones Determine Immune Response. Front Immunol. 2018; 9: 1931.

3. Stevanato Filho PR, Aguiar Júnior S, Begnami MD, et al. Estrogen Receptor $\beta$ as a Prognostic Marker of Tumor Progression in Colorectal Cancer with Familial Adenomatous Polyposis and Sporadic Polyps. Pathol Oncol Res. 2018; 24: 533-40.

4. Rajabi P, Bagheri M, Hani M. Expression of Estrogen Receptor Alpha in Malignant Melanoma. Adv Biomed Res. 2017; 6: 14

5. de Giorgi V, Mavilia C, Massi D, et al. Estrogen receptor expression in cutaneous melanoma: a real-time reverse transcriptase-polymerase chain reaction and immunohistochemical study. Arch Dermatol. 2009; 145: 30-6.

6. Mori T, Martinez SR, O'Day SJ, et al. Estrogen receptor-alpha methylation predicts melanoma progression. Cancer Res. 2006; 66: 6692-8.

7. de Giorgi V, Gori A, Gandini S, et al. Oestrogen receptor beta and melanoma: a comparative study. Br J Dermatol. 2013; 168: 513-9.

8. Udovicic-Gagula D, Ahmovic A, Bilalovic N, et al. Expression of Ki-67 and Estrogen Receptor Beta in Primary Cutaneous Melanoma as a Potential Indicator of Regional Lymph Node Positivity. Appl Immunohistochem Mol Morphol. 2019; 27: 27-32.

9. Gupta S, Artomov M, Goggins W, et al. Gender Disparity and Mutation Burden in Metastatic Melanoma. J Natl Cancer Inst. 2015; 107: djv221.

10. Gutiérrez-Castañeda LD, Nova JA, Tovar-Parra JD. Frequency of mutations in BRAF, NRAS, and KIT in different populations and histological subtypes of melanoma: a systemic review. Melanoma Res. 2020; 30: 62-70.

11. Muñoz-Couselo E, Adelantado EZ, Ortiz C, et al. NRAS-mutant melanoma: current challenges and future prospect. Onco Targets Ther. 2017; 10: 3941-7.

12. Tanda ET, Vanni I, Boutros A, et al. Current State of Target Treatment in BRAF Mutated Melanoma. Front Mol Biosci. 2020; 7: 154.

13. Pavlick AC, Zhao R, Lee C-H, et al. First-line immunotherapy versus targeted therapy in patients with BRAF-mutant advanced melanoma: a real-world analysis. Future Oncol. 2021; 17: 689-99.

14. Warburton L, Meniawy TM, Calapre L, et al. Stopping targeted therapy for complete responders in advanced BRAF mutant melanoma. Sci Rep. 2020; 10: 18878.

15. Delyon J, Lebbe C, Dumaz N. Targeted therapies in melanoma beyond BRAF: targeting NRAS-mutated and KIT-mutated melanoma. Curr Opin Oncol. 2020; 32: 79-84.

16. Petrova V, Arkhypov I, Weber R, et al. Modern Aspects of Immunotherapy with Checkpoint Inhibitors in Melanoma. Int J Mol Sci. 2020; 21: 2367.

17. Zimmer L, Livingstone E, Hassel JC, et al. Adjuvant nivolumab plus ipilimumab or nivolumab monotherapy versus placebo in patients with resected stage IV melanoma with no evidence of disease (IMMUNED): a randomised, double-blind, placebo-controlled, phase 2 trial. Lancet 2020; 395: 1558-68.

18. Bellenghi M, Puglisi R, Pontecorvi G, et al. Sex and Gender Disparities in Melanoma. Cancers (Basel) 2020; 12: 1819.

19. Zhao L, Huang S, Mei S, et al. Pharmacological activation of estrogen receptor beta augments innate immunity to suppress cancer metastasis. Proc Natl Acad Sci U S A. 2018; 115: E3673-81.

20. Sareddy GR, Li X, Liu J, et al. Selective Estrogen Receptor $\beta$ Agonist LY500307 as a Novel Therapeutic Agent for Glioblastoma. Sci Rep. 2016; 6: 24185.

21. Reese JM, Bruinsma ES, Monroe DG, et al. ER $\beta$ inhibits cyclin dependent kinases 1 and 7 in triple negative breast cancer. Oncotarget 2017; 8: 96506-21.

22. Bellenghi M, Puglisi R, Pedini F, et al. SCD5-induced oleic acid production reduces melanoma malignancy by intracellular retention of SPARC and cathepsin B. J Pathol. 2015; 236: 315-25.

23. Puglisi R, Bellenghi M, Pontecorvi G, et al. SCD5 restored expression favors differentiation and epithelial-mesenchymal reversion in advanced melanoma. Oncotarget 2018; 9: 7567-81.

24. Anichini A, Mazzocchi A, Fossati G, et al. Cytotoxic T lymphocyte clones from peripheral blood and from tumor site detect intratumor heterogeneity of melanoma cells. Analysis of specificity and mechanisms of interaction. J Immunol. 1989; 142: 3692-701.

25. Scuderi N, Onesti MG, Bistoni G, et al. The clinical application of autologous bioengineered skin based on a hyaluronic acid scaffold. Biomaterials. 2008; 29: $1620-9$.

26. Genomic Data Commons data portal. https://portal.gdc.cancer.gov.

27. Colaprico A, Silva TC, Olsen C, et al. TCGAbiolinks: an R/Bioconductor package for integrative analysis of TCGA data. Nucleic Acids Res. 2016; 44: e71.

28. Robinson MD, McCarthy DJ, Smyth GK, edgeR: a Bioconductor package for differential expression analysis of digital gene expression data. Bioinformatics 2010; 26: 139-40.

29. Felicetti F, Errico MC, Bottero L, et al. The promyelocytic leukemia zinc finger-microRNA-221/-222 pathway controls melanoma progression through multiple oncogenic mechanisms. Cancer Res. 2008; 68: 2745-54.

30. Compton DR, Sheng S, Carlson KE, et al. Pyrazolo[1,5-a]pyrimidines: estrogen receptor ligands possessing estrogen receptor beta antagonist activity. J Med Chem. 2004; 47: 5872-93.

31. Sun J, Huang YR, Harrington WR, et al. Antagonists selective for estrogen receptor alpha. Endocrinology 2002; 143: 941-7. 
32. Otto, T., Sicinski, P. Cell cycle proteins as promising targets in cancer therapy. Nat Rev Cancer. 2017; 17: 93-115.

33. Choi EK, Lim JA, Kim JK, et al. Cyclin B1 stability is increased by interaction with BRCA1, and its overexpression suppresses the progression of BRCA1-associated mammary tumors. Exp Mol Med. 2018; 50: 1-16.

34. Chang DC, Xu N, Luo KQ. Degradation of cyclin B is required for the onset of anaphase in Mammalian cells. J Biol Chem. 2003; 278: 37865-73.

35. Matheson CJ, Backos DS, Reigan P. Targeting WEE1 Kinase in Cancer. Trends Pharmacol Sci. 2016; 37: 872-81.

36. Podhorecka M, Skladanowski A, Bozko P. H2AX Phosphorylation: Its Role in DNA Damage Response and Cancer Therapy. J Nucleic Acids. 2010; 2010: 920161.

37. Krakhmal NV, Zavyalova MV, Denisov EV, et al. Cancer Invasion: Patterns and Mechanisms. Acta Naturae. 2015; 7: 17-28.

38. Zhou Y, Liu X. The role of estrogen receptor beta in breast cancer. Biomark Res. 2020; 8: 39 .

39. Di Zazzo E, Galasso G, Giovannelli P, et al. Estrogen Receptors in Epithelial-Mesenchymal Transition of Prostate Cancer. Cancers (Basel) 2019; 11: 1418.

40. Pearlman RL, Montes de Oca MK, Pal HC, et al. Potential therapeutic targets of epithelial-mesenchymal transition in melanoma. Cancer Lett. 2017; 391: 125-40.

41. Mal R, Magner A, David J, et al. Estrogen Receptor Beta (ER $\beta$ ): A Ligand Activated Tumor Suppressor. Front Oncol. 2020; 10: 587386.

42. Liu M, Du Y, Li H, et al. Cyanidin-3-o-Glucoside Pharmacologically Inhibits Tumorigenesis via Estrogen Receptor $\beta$ in Melanoma Mice. Front Oncol. 2019; 9: 1110 .

43. Glatthaar H, Katto J, Vogt T, et al. Estrogen Receptor Alpha (ESR1) Single-Nucleotide Polymorphisms (SNPs) Affect Malignant Melanoma Susceptibility and Disease Course. Genet Epigenet. 2016; 8: 1-6.

44. Huang S, Zhou N, Zhao L, et al. Pharmacological Activation of Estrogen Receptor Beta Overcomes Tumor Resistance to Immune Checkpoint Blockade Therapy. iScience 2020; 23: 101458.

45. Yan S, Dey P, Ziegler Y, et al. Contrasting activities of estrogen receptor beta isoforms in triple negative breast cancer. Breast Cancer Res Treat. 2021; 185: 281-92. 